\title{
HAUSDORFF-ANALYTIC FUNCTIONS OF MATRICES ${ }^{1}$
}

\author{
WALTER O. PORTMANN ${ }^{2}$
}

1. Introduction. The concept of Hausdorff-analytic ( $H$-analytic) functions on an (associative) hypercomplex system, over the complex number field, with an identity [1] is, in this paper, applied to functions of matrices with distinct eigenvalues. In the process of showing a sufficient condition for a matric function to be $H$-analytic in a neighborhood of a matrix $Z_{0}$ with distinct eigenvalues, it is shown that the projections (Frobenius covariants) are $H$-analytic in a neighborhood of $Z_{0}$, and that there exists a matric function $Q=Q(Z)$ which is $H$-analytic in a neighborhood of $Z_{0}$ such that $Q^{-1} Z Q=\Lambda=\operatorname{diag}\left(\lambda_{k}\right)$, $\lambda_{i} \neq \lambda_{j}$ for $i \neq j$, for all $Z$ in that neighborhood of $Z_{0}$.

It has previously been shown [2] that if $f(Z)$ is a function on 9 (the algebra of all square matrices of order $n$ over the complex number field) whose component functions are analytic functions, in some open domain, of the complex (component) variables $z_{i j}$ of $Z=\left(z_{i j}\right)$, then $f(Z)$ is $H$-analytic in a corresponding open domain of $\mathfrak{T}$.

2. $H$-analyticity of the projections of a matrix with distinct eigenvalues.

Lemma 2.1. The projections (or Frobenius covariants) $P_{k}(Z)$ corresponding to the eigenvalues $\lambda_{k}, k=1, \cdots, s$, of a matrix $Z$ in IT are given by

$$
P_{k}(Z)=\frac{1}{2 \pi i} \int_{C_{k}}(\lambda I-Z)^{-1} d \lambda,
$$

where $C_{k}$ is a circle in the complex $\lambda$-plane containing $\lambda_{k}$ but none of the other $\lambda_{j}$.

Proof. By [4, p. 22],

$$
(\lambda I-Z)^{-1}=\sum_{j=1}^{s} P_{j}(Z) \sum_{m=0}^{r_{j}-1} \frac{\left(Z-\lambda_{j} I\right)^{m}}{\left(\lambda-\lambda_{j}\right)^{m+1}}
$$

where $r_{j}$ is the index of $\lambda_{j}$.

The integral, over a curve in the complex $\lambda$-plane, of a matrix of complex functions is given by the matrix of integrals, over the curve, of the matric elements. Therefore if we let $C_{k}$ be a circle containing

Received by the editors January 13, 1959 and, in revised form, April 13, 1959.

1 This paper was prepared under the facilities granted by the Case Research Fund.

2 The author is now associated with Arizona State University. 
$\lambda_{k}$ but none of the other $\lambda_{j}$, then

$$
\begin{aligned}
\frac{1}{2 \pi i} \int_{C_{k}}(\lambda I- & Z)^{-1} d \lambda \\
& =\frac{1}{2 \pi i} \sum_{j=1}^{s} P_{j}(Z) \sum_{m=0}^{r_{j}-1}\left(Z-\lambda_{j} I\right)^{m} \int_{C_{k}} \frac{d \lambda}{\left(\lambda-\lambda_{j}\right)^{m+1}}=P_{k}(Z) .
\end{aligned}
$$

We will use this representation to prove the following.

THEOREM 2.1. If $Z_{0}$ is a matrix with distinct eigenvalues, then there exists a neighborhood $N$ of $Z_{0}$ such that for $Z$ in $N, P_{k}(Z)$ is an $H$ analytic function of $Z$.

Proof. Let $\lambda_{k}^{0}, k=1, \cdots, n$, be the (distinct) eigenvalues of $Z_{0}$, then

$$
P_{k}\left(Z_{0}\right)=\frac{1}{2 \pi i} \int_{C_{k}}(\lambda I-Z)^{-1} d \lambda
$$

is the projection of $Z_{0}$ corresponding to $\lambda_{k}^{0}$, where $C_{k}$ is a sufficiently small circle which has $\lambda_{k}^{0}$ as its center and all other $\lambda_{j}^{0}$ in its exterior, that is, if $\left|\lambda_{k}^{0}-\lambda_{j}^{0}\right|>2 \epsilon$ for $j \neq k$, then $C_{k}:\left|\lambda-\lambda_{k}^{0}\right|=\epsilon$ will be sufficient.

Now, for all matrices $Z$ sufficiently near $Z_{0}$, that is, such that $\operatorname{norm}\left(Z-Z_{0}\right)$ is sufficiently small (where, for convenience, the norm of any matrix $X=\left(x_{i j}\right), i=1, \cdots, m, j=1, \cdots, n$, with complex components, shall be defined by $\left.\operatorname{norm}(X)=\max _{i, j}\left|x_{i j}\right|\right)$, the eigenvalues $\lambda_{1}, \cdots, \lambda_{n}$ of $Z$ will be near those of $Z_{0}$, since the eigenvalues of a matrix are continuous functions of the elements of the matrix [2]. Thus, a neighborhood $N$ of $Z_{0}$ may be chosen such that $\left|\lambda_{k}-\lambda_{k}^{0}\right|$ $<\epsilon, k=1, \cdots, n$, then for each $Z$ in $N, Z$ has distinct eigenvalues $\lambda_{j}$, and $\lambda_{k}$ lies within $C_{k}$ while all other $\lambda_{j}$ lie outside $C_{k}$. Hence for all $Z$ in $N$,

$$
P_{k}(Z)=\frac{1}{2 \pi i} \int_{C_{k}}(\lambda I-Z)^{-1} d \lambda .
$$

The $r, s$ element of the matrix $P_{k}(Z)$ is given by

$$
P_{k}(Z)_{r s}=\frac{1}{2 \pi i} \int_{C_{k}} R_{r s}\left(\lambda, z_{i j}\right) d \lambda,
$$

where $R_{r s}\left(\lambda, z_{i j}\right)$ is the quotient of two polynomials in $\lambda$ and the $z_{i j}$, $i, j=1, \cdots, n$. Since $C_{k}$ does not pass through any of the zeros of $\operatorname{det}(\lambda I-Z)$, regardless of what $Z$ in $N$ is chosen, $R_{r s}\left(\lambda, z_{i j}\right)$ is a concontinuous function of the complex variables $\lambda$ and $z_{i j}, i, j=1, \cdots, n$, 
where each $z_{i j}$ ranges over a region $N_{i j}$ determined by $N$, and $\lambda$ lies on $C_{k}$; also $R_{r s}\left(\lambda, z_{i j}\right)$ is an analytic function of each $z_{i j}$ in $N_{i j}$, for every value of $\lambda$ of $C_{k}$. Therefore, $P_{k}(Z)_{r s}$ is an analytic function of each $z_{i j}$ of $Z$ in $N$, and hence, the components $P_{k}(Z)_{r s}$ are analytic functions of $z_{i j}$, that is $P_{k}(Z)$ is $H$-analytic at $Z$ in $N$.

\section{The existence of $Q(Z)$.}

LEMMA 3.1. If $Z_{0}$ is a matrix with distinct eigenvalues and $x$ is a vector such that $P_{k}\left(Z_{0}\right) x \neq 0$ for every $k=1, \cdots, n$, then for $Z$ sufficiently near $Z_{0}, P_{k}(Z) x \neq 0, k=1, \cdots, n$.

Proof. Since $P_{k}\left(Z_{0}\right) x \neq 0$ for every $k=1, \cdots, n$, there exists a $\delta>0$ such that norm $\left(P_{k}\left(Z_{0}\right) x\right)>\delta$ for every $k$. Now $P_{k}(Z)$ is an $H$ analytic function of $Z$ in a neighborhood of $Z_{0}$ and therefore a continuous function of $Z$ in a neighborhood of $Z_{0}$. Thus, for $Z$ near $Z_{0}$, $P_{k}(Z)$ is near $P_{k}\left(Z_{0}\right)$ and therefore $P_{k}(Z) x$ is near $P_{k}\left(Z_{0}\right) x$; in particular $Z$ may be chosen sufficiently close to $Z_{0}$ such that

$$
\operatorname{norm}\left(P_{k}\left(Z_{0}\right) x-P_{k}(Z) x\right)<\delta / 2,
$$

for all $k$. Hence, for all $Z$ in such a neighborhood $N$ of $Z_{0}$,

$$
\begin{aligned}
\operatorname{norm}\left(P_{k}(Z) x\right) & \geqq \operatorname{norm}\left(P_{k}\left(Z_{0}\right) x\right)-\operatorname{norm}\left(P_{k}\left(Z_{0}\right) x-P_{k}(Z) x\right) \\
& >\delta-\delta / 2=\delta / 2>0 .
\end{aligned}
$$

Thus, for $Z$ in $N, P_{k}(Z) x \neq 0$ for every $k=1, \cdots, n$.

ThEOREM 3.1. Let $Z_{0}$ be a matrix with distinct eigenvalues; then there exists a nonsingular matrix $Q$ whose components are analytic functions of the elements of $Z$, for $Z$ in some neighborhood $N$ of $Z_{0}$ (therefore $Q$ and $Q^{-1}$ are $H$-analytic functions of $Z$ in $\left.N\right)$, such that $Q^{-1} Z Q=\Lambda=\operatorname{diag}\left(\lambda_{k}\right)$ for all $Z$ in $N$.

Proof. Choose a vector $x$ such that $P_{k}\left(Z_{0}\right) x \neq 0$ for all $k=1, \cdots, n$, then by Lemma 3.1 , for $Z$ sufficiently near $Z_{0}, Q_{k}=P_{k}(Z) x \neq 0$. By $\left[4\right.$, p. 22], $\left(Z-\lambda_{k} I\right) Q_{k}=0$, since the index $r_{k}$ of $\lambda_{k}$ is 1 for all $k$, that is, $Z Q_{k}=\lambda_{k} Q_{k}$. Now, let $Q$ be the matrix whose $j$ th column is $Q_{j}$, then $Z Q=Q \Lambda$, where $\Lambda=\operatorname{diag}\left(\lambda_{k}\right)$. Therefore, since the $Q_{k}$ are linearly independent $Q^{-1} Z Q=\Lambda$.

Since $Q_{k}=P_{k}(Z) x$, the components of $Q_{k}$ are linear combinations of the elements of $P_{k}(Z)$ and therefore, by Theorem 2.1 , they are analytic functions of the elements $z_{i j}$ of $Z$ in a neighborhood of $Z_{0}$. Hence $Q$ is $H$-analytic in a sufficiently small neighborhood $N$ of $Z_{0}$.

Also $Q^{-1}=\left(S_{i j} / \operatorname{det}(Q)\right)$, where $S_{i j}$ is the cofactor of the $j, i$ component of $Q$. Since $\operatorname{det}(Q) \neq 0$, and $S_{i j}$ and $\operatorname{det}(Q)$ are polynomials 
with constant coefficients in the analytic components of $Q$, the components at $Q^{-1}$ are analytic functions of the elements of $Z$ in $N$ and therefore $Q^{-1}$ is also $H$-analytic in $N$.

Note: If one knows a $Q_{0}$ such that $Q_{0}^{-1} Z_{c} Q_{0}=\Lambda_{0}=\operatorname{diag}\left(\lambda_{k}^{0}\right)$ for the given $Z_{0}$, then, for the above $x$, one may choose $x=\sum_{j=1}^{n} Q_{0}^{(j)}$, where $Q_{0}^{(j)}$ is the $j$ th column of $Q_{0}$, since $Z_{0} Q_{0}^{(k)}=\lambda_{k}^{0} Q_{0}^{(k)}$, that is $P_{k}\left(Z_{0}\right) Q_{0}^{(j)}$ $=\delta_{j k} Q_{0}^{(k)}$, and therefore $P_{k}\left(Z_{0}\right) x=Q_{0}^{(k)} \neq 0$ for every $k=1, \cdots, n$.

Corollary 3.1. If the matrix $Z$ has distinct eigenvalues $\lambda_{k}, k$ $=1, \cdots, n$, then the $\lambda_{k}$ are analytic functions of the components $z_{i j}$ of $Z$.

4. A sufficient condition for $H$-analyticity of a matric function at a matrix with distinct eigenvalues. The hypothesis of (i) of the following theorem would be desirable if one wished to view this as a theory for functions of linear transformations of a finite dimensional vector space, for the invariance under similarity transformations, $F(Y)$ $=P^{-1} F(X) P$ for $Y=P^{-1} X P$, permits the definition of a function of a finite dimensional linear transformation to be independent of the choice of basis for the vector space.

THEOREM 4.1. Let $F=\sum_{i, j=1}^{n} f_{i j} E_{i j}$ be a matric function (where $E_{i j}$ is the $n \times n$ matrix with $a 1$ in the $i, j$ position and zeros elsewhere).

(i) Let $F$ be such that, $F$ defined at $X$ and $Y=P^{-1} X P$ implies that $F$ is defined at $Y$ and $F(Y)=P^{-1} F(X) P$; then, for a given $X$ at which $F$ is defined, $F(X)$ is a polynomial in $X$. In particular, if $\Lambda=\operatorname{diag}\left(\lambda_{i}\right)$ is a diagonal matrix at which $F$ is defined, then $F(\Lambda)$ is a diagonal matrix, that is,

$$
\left.f_{i j}\right]_{z_{r s}=\delta_{r s} \lambda_{r}}=\delta_{i j} g_{i}\left(\lambda_{1}, \cdots, \lambda_{n}\right) .
$$

(ii) Further, if $F$ is also such that the diagonal component functions $f_{i i}$ are analytic functions of each $\lambda_{j}$ at the components of a diagonal matrix with distant eigenvalues $\lambda_{i}$, at which $F$ is defined, that is,

$$
\left.\frac{\partial f_{i i}}{\partial z_{j j}}\right]_{z_{r g}=\delta_{r s} \lambda_{r}}=\frac{\partial g_{i}}{\partial \lambda_{j}}
$$

exist for $i, j=1, \cdots, n$, then, $F$ is $H$-analytic at a matrix $Z$ with distinct eigenvalues at which $F$ is defined.

Proof. To prove the first part of this theorem we shall use the following lemma obtained by Richter [3].

LEMma 4.1. Let $F$ be a function which satisfies the hypothesis of (i) of Theorem 4.1, and let $X$ be a matrix for which $F(X)$ is defined. If 
$X B=B X$, then $F(X) B=B F(X)$, that is, $F(X)$ commutes with every matrix which commutes with $X$.

Any matrix $F(X)$ satisfying the conclusion of the above lemma is a polynomial in $X[5]$.

To prove the second part of the theorem, let $F$ be defined at a matrix $Z$ with distinct eigenvalues. By Theorem 3.1 there exists a $Q=Q(Z), H$-analytic at $Z$, such that $Q^{-1} Z Q=\Lambda=\operatorname{diag}\left(\lambda_{i}\right)$, where the $\lambda_{i}$ are the eigenvalues of $Z$; therefore by (i), $F(Z)=Q F(\Lambda) Q^{-1}$ and

$$
F(\Lambda)=\sum_{i=1}^{n} g_{i} E_{i i} .
$$

Therefore, since the $g_{i}$ are analytic functions of the $\lambda_{j}$ and by Corollary 3.1, the $\lambda_{j}$ are analytic functions of the $z_{r s}, r, s=1, \cdots, n$, the $f_{i j}$ are analytic functions (in a neighborhood) of the components $z_{r s}$ of $Z$. Thus $F$ if $H$-analytic at $Z$.

\section{REFERENCES}

1. F. Hausdorff, Zur Theorie der Systeme complexer Zahlen, Leipziger Berichte vol. 52 (1900) pp. 43-61.

2. W. O. Portmann, A derivative for Hausdorff-analytic functions, Proc. Amer. Math. Soc. vol. 10 (1959) pp. 101-105.

3. H. Richter, Uber Matrixfunktionen, Math. Ann. vol. 122 (1950) pp. 16-34.

4. H. Schwerdtfeger, Les fonctions de matrices, Les Fonctions Univalentes, Actualités Sci. Ind., no. 649, Paris, Hermann and Co., 1938.

5. H. W. Turnbull and A. C. Aitken, An iniroduction to the theory of canonical matrices, Blackie and Son Ltd., 1932, Chap. X.

Case Institute of Technology 